\title{
A Successful Broker Agent for Power TAC
}

\author{
Bart Liefers ${ }^{(凶)}$, Jasper Hoogland, and Han La Poutré \\ Centrum Wiskunde and Informatica (CWI), P.O. Box 94079, \\ 1090 GB Amsterdam, The Netherlands \\ liefers@cwi.nl
}

\begin{abstract}
The Power TAC simulates a smart grid energy market. In this simulation, broker agents compete for customers on a tariff market and trade energy on a wholesale market. It provides a platform for testing strategies of broker agents against other strategies. In this paper we describe the strategies of our broker agent. Amongst others, due to a beneficial trading technique related to equilibria in continuous auctions on the wholesale market and a strategy inspired by Tit-for-Tat in the Iterated Prisoner's Dilemma game on the tariff market, our broker ended second in the 2013 Power TAC.
\end{abstract}

\section{Introduction}

Variable prices for electrical energy are seen as an important prerequisite for the development of smart grids [1]. These prices can provide an economical motivation for consumers to shift part of their loads from peak times to times where energy is more abundant. Various mechanisms for setting prices for electricity in the retail market are proposed [2]. The performance of these mechanisms depends on the behaviour of self-interested actors, and they should therefore be tested in a competitive setting.

The Power Trading Agent Competition (Power TAC) is a platform in which agent strategies for trading energy in a smart grid setting can be tested. Competing researchers create agents (referred to as 'brokers') that act in the retail market for electricity. They try to maximize their profit by publishing tariffs to attract customers and by trading on a wholesale market. After a pilot and a demonstration in 2011 and 2012, the inaugural Power TAC was held in 2013 [3].

In this paper we present cwiBroker, a broker that was created for this competition. It utilized an adaptive strategy in order to achieve the most profitable setting for itself. Thus, the actions our broker takes depend on the actions of other brokers. In the tariff market, we took into account the number of competitors, which yielded strategies suitable for either a duopoly or an oligopoly setting. In duopoly games, our broker uses an adaptive strategy inspired by Tit-for-Tat in the Iterated Prisoner's Dilemma [4] game. In oligopoly games, our broker aims to find the optimal tariff by estimating the profit for a set of candidate tariffs. Furthermore, we used regression analysis to accurately make predictions about energy prosumption. Finally, for wholesale energy we used a beneficial 
trading technique, related to equilibria in continuous auctions. The combined set of strategies of cwiBroker resulted in a second place in the competition.

This document is organised as follows. In Sect. 2 we describe our broker's basic architecture and the setting in which it has to act. Next, each of the components of our broker will be described in more detail (Sect. 3 for the tariff market, Sect. 4 for the wholesale market, and Sect. 5 for the prosumption estimator). In Sect. 6 we will analyse the performance of each component, based on results of the 2013 Power TAC.

\section{The 2013 Power Trading Agent Competition}

In the Power TAC [3], a central server simulates several entities that act in a smart grid environment. Among those entities are large generation companies (gencos), consumers (e.g. households, offices, hospitals), small producers (e.g. owners of solar panels and wind farms), a distribution utility (DU), and a wholesale market. Brokers that trade in this environment are created by competing researchers. The brokers are tested against each other in various settings (differing in e.g. number of players, weather conditions or customer behavior).

In the simulations, time is divided into 1-h intervals, referred to as timeslots. Within each timeslot, demand and supply must be balanced. This means the net amount of energy bought and sold on different markets must be zero. Three different markets are present: a tariff market, a wholesale market and a balancing market. On the tariff market brokers trade energy with consumers and small producers by publishing consumption and production tariffs respectively. Potential customers evaluate the tariffs and subscribe to the one they prefer. On the wholesale market energy is traded in a periodic double-sided auction, in which the brokers can trade energy ahead with each other and with the gencos. If a broker has a real-time net imbalance, then the DU will buy or sell energy for the broker in order to match demand and supply on the balancing market. The rewards or penalties for imbalances depend on the imbalance of the broker, but also on the total imbalance of all brokers. In addition to the competing brokers, in every game there is a default broker, to which all customers are initially subscribed. For a detailed description of Power TAC we refer to [3].

In the 2013 Power TAC, there were 7 participants. Each broker played six 2-player games (one against each competitor) and 20 4-player games (one for each combination of three out of the six other players). Four games with all 7 participants were held. Each game consists of at least 1320 timeslots. After that, there is a fixed probability that the game will end in each timeslot.

The cwiBroker. Participation in the Power TAC requires brokers to make complex decisions. Roughly speaking, brokers have to operate on two different markets: the tariff market and the wholesale market. The balancing market could theoretically also be used strategically, but this is hard, and penalties for under supply are generally higher than rewards for over supply [5]. Our broker, called cwiBroker, therefore aims to balance its own demand and supply. 
In order to achieve this, we created a component that estimates the production or consumption of each type of customer. Furthermore, we created separate components for the tariff market and the wholesale market.

Although the task for each component is quite complex, their interaction is rather straightforward. The prosumption estimator requires as input the historical data (energy usage of each type of customer and weather data). Given a weather forecast, it predicts the production or consumption for a future timeslot for each type of customer. This is used as input for the wholesale market component. The latter trades on the wholesale market in order to balance the estimated prosumption for cwiBroker and maintains, for each quantity, an expected unit price for wholesale energy (based on historical results). This expected unit price is used as input for the tariff market component, which uses this information to decide which tariffs will be most profitable.

\section{$3 \quad$ Tariff Market Strategy}

In this section we will describe the tariff market component, which manages the set of published tariffs. It can publish new tariffs, modify existing tariffs, or revoke old tariffs. A tariff consists of rates, which specify a retail price that may depend on the time of the day, day of the week, or the quantity that is consumed or produced. A tariff may also contain features such as a periodic fee, a sign up bonus or fee, or an early-exit fee. The number of customers that a tariff attracts, depends on its exact specification and the alternative tariffs that are available.

The optimal strategy for trading on the tariff market depends on the behavior of other brokers, in particular the tariffs they publish. Therefore, our broker compares its tariffs with the tariffs of opponents. The structure of our broker's tariffs is kept relatively simple. It publishes at most one tariff at a time, with a fixed-price, single-rate, no sign-up bonuses or fee's, no early-exit bonuses, and no periodic payments. It can therefore be represented by a single price $p$. We will make some simplifications when considering the tariffs of opponents. When opponents publish multiple tariffs, we will only consider their cheapest tariff. This tariff is expected to be the most relevant one, since it attracts the most customers. Furthermore, we take for any tariff only a single price, equal to the lowest rate in the tariff. The more complex features of a tariff are ignored.

We argue that, in order to obtain high revenues, brokers should cooperate with each other. This can be achieved if all brokers publish tariffs that are very profitable to them, but unfavourable for customers. In this case, the brokers each obtain a similar share of customers, and may make large revenues. However, if one broker does not cooperate, by publishing more competitive tariffs, it will attract more customers than other brokers, which may trigger competition between them. This competition may lead to a price war, in which tariff prices ultimately approach the cost price for brokers. In this case it will be very hard for brokers to make a profit. It is possible to avoid this situation if all brokers cooperate. However, if one broker does not cooperate, this can be sufficient to make cooperation of other brokers fail. For this reason, cooperation is more likely 
to succeed in games with fewer brokers. Therefore, we implemented different tariff market strategies for duopoly games (games with two players) and oligopoly games (with three or more players).

\subsection{The Duopoly Tariff Market Strategy}

In duopoly games, our broker is initially competitive, i.e. it first tries to obtain a reasonable number of customers. However, we do not always want to price cut our opponent, if this leads to unprofitable tariffs. Therefore, after we published a number of competitive tariffs, we switch to a strategy in which we copy the tariffs of the opponent. In case our opponent also no longer competes with us, we can achieve a tacit collusion. If our opponent reacts by increasing its prices, we also copy the higher prices. In this case both brokers may make large revenues.

In more detail, our broker publishes an initial tariff with a relatively low price $p_{\text {min }}$, which is chosen to be higher than the estimated cost price. After that, whenever the opponent publishes a tariff with price $p^{\prime}$, our agent responds by replacing its current tariff with a new tariff with price $p=p^{\prime}-\Delta$, within bounds $\left[p_{\min }, p_{\max }\right]$. First we choose $\Delta>0$, but after two times we use $\Delta=0$.

Intuitively, our strategy has some analogies with Tit-for-Tat (TFT) in the Iterated Prisoner's Dilemma (IPD) [4]. The duopoly game is somewhat similar to IPD, in the sense that brokers have an incentive to compete with their opponent (the 'Defect' action in IPD), but their profits would be higher if both published high-priced tariffs (the 'Cooperate' action in IPD). After the first few competitive actions, our broker will copy the last action from the opponent in the rest of the game. This is analogous to TFT.

\subsection{The Oligopoly Tariff Market Strategy}

We will now describe our broker's tariff market strategy in oligopoly games. In oligopoly games, a cooperative strategy is less likely to succeed than in duopoly games, so in these games our broker plays a more competitive strategy. Our broker considers a set of tariff prices and estimates for each price $p$ in this set how much profit it would make if it published a tariff this price. It then publishes the most profitable one. Our broker computes an estimation $\hat{T P}(p)$ of the profit it would make if it published a tariff with retail price $p$ :

$$
\hat{T P}(p)=\left(p-\hat{p}_{\text {cost }}\right) \sum_{c \in C} \hat{N}_{c}(p) \hat{q}_{c}
$$

where $C$ is the set of customer types and $\hat{p}_{\text {cost }}$ is the estimated cost price of energy. $\hat{q}_{c}$ is the estimated prosumption per customer of type $c$, computed by the prosumption estimator (see Sect. 5$). \hat{N}_{c}(p)$ is the estimated number of customers of type $c$ that subscribe to a tariff with price $p$. It is modelled as:

$$
\hat{N}_{c}(p)=N_{c}^{t o t a l} \frac{e^{b p}}{a+e^{b p}}
$$


The total number of consumers $N_{c}^{\text {total }}$ of type $c$ in the game is public information. The parameters $a$ and $b$ are estimated during the game. This is done by sequentially publishing two test tariffs with different prices $p_{1}, p_{2}$. The broker keeps each tariff for a number of timeslots and records the number of customers $N_{c, 1}$ and $N_{c, 2}$ respectively, for each customer type $c$. The parameters $a, b$ are then computed from the pairs $\left\langle p_{1}, N_{c, 1}\right\rangle,\left\langle p_{2}, N_{c, 2}\right\rangle$. Finally, our broker computes $\hat{T P}(p)$ for several $p$, and publishes a tariff with the price $p$ that maximizes $\hat{T P}(p)$.

In order to successfully estimate $\hat{N}_{c}(p)$, the other brokers must not publish tariffs while $N_{c, 1}, N_{c, 2}$ are being measured. Furthermore, even if $\hat{N}_{c}(p)$ has been successfully determined, the computed optimal tariff price only remains optimal as long as other brokers publish no new tariffs. Thus, this approach only works if the set of published tariffs remains stable. Due to the fierce competition in oligopoly games, we expect tariff prices to reach an equilibrium quickly. Therefore, our broker waits a period of time before it starts estimating the subscription models. After a predetermined number of timeslots $K_{1}$, it publishes a competitive tariff with a price $p_{\text {init }}$ equal to the lowest observed price of competitors. Then, after $K_{2}$ more timeslots, it will publish both test tariffs sequentially, each for $K_{3}$ timeslots. Finally, it publishes the estimated optimal tariff.

If the subscription models are inaccurate, or if the environment changes because other brokers have published new tariffs, then the estimated optimal tariff may not be correct (anymore). In order to escape from such situations, our broker aims for a number of customers $N$ of the type CentervilleHomes within a manually chosen interval $\left[N_{\min }, N_{\max }\right]$. If $N<N_{\min }$, then our broker's current tariff (with price $p$ ) is too expensive and it will be replaced by a new tariff with a lower price $p^{\prime}=p-\delta$. If $N>N_{\text {max }}$, then our broker's tariff (with price $p$ ) is too cheap and it will be replaced by a new tariff with a higher price $p^{\prime}=p+\delta$. $\delta$ is chosen manually. Our broker keeps replacing tariffs as long as $N \notin\left[N_{\min }, N_{\max }\right]$. We expect that if a tariff attracts customers for type CentervilleHomes, it is likely to attract customers of other types too.

\section{The Wholesale Market Strategy}

In this section we will describe the strategy played by cwiBroker on the wholesale market. We will first introduce the market rules and properties of other players (in particular the gencos) and analyse the particularities of the Power TAC wholesale market design. Next, we explain the strategies cwiBroker used to obtain the desired amount of energy at a unit price that is most profitable.

\subsection{Power TAC Wholesale Market Properties}

On the Power TAC wholesale market, energy for a particular timeslot can be traded in 24 sequential auctions (between one and 24 timeslots ahead). Brokers can place both bids (orders to buy energy) and asks (orders to sell energy), represented by a quantity and an optional limit price. This limit price represents a maximum price to buy or a minimum price to sell. There is no official limit on 
the number of orders a broker is allowed to submit for a single auction (although there is a practical limit due to overhead for the server).

The market is cleared as follows. Bids are sorted in descending order of limit price, and asks in ascending order. The orders will be cleared iteratively, as long as the limit price of the first bid is higher than the limit price of the first ask. If the quantities $q$ of the cleared orders are not equal, the quantity $\min \left(q_{b i d}, q_{a s k}\right)$ will be cleared, and the remainder of either the bid or the ask will be kept for the next iteration. If the ask price exceeds the bid price, or if there are no more bids or asks left, this process will terminate. The market clearing price is set at the mean of the last cleared ask price and the last cleared bid price.

Besides the orders of the brokers, the gencos send in asks for their capacity (which is variable, determined by a mean reverting random walk around their nominal capacity) for a fixed limit price. If a genco has already sold a part of its capacity in previous auctions for a specific timeslot, it will send in an ask for the remainder in the next auction, at the same limit price (which is constant for a single genco, but varies between them).

\subsection{Strategy}

The particular market design chosen to represent the wholesale market in Power TAC can be regarded as a mix between a call market and a continuous market. Each individual market clearing is a call market, because orders of all brokers are gathered and cleared once per timeslot. However, this process is repeated 24 times for each timeslot. Traders are thus flexible in choosing when to place their orders, like in a continuous market.

It is not uncommon for continuous auctions to open the trading with a call market [6]. The opening call market will yield an equilibrium price and quantity for the specific commodity that is traded. Any possible future deviations will be traded in the subsequent continuous auction. In the Power TAC wholesale market, brokers should therefore try to trade their desired amounts of energy in the first auction for each timeslot. The subsequent auctions facilitate trading of fluctuations in demand or supply due to e.g. changes in predicted number of customers or weather forecasts.

In contrast to this analysis, it may be tempting for brokers to start bidding at relatively low prices in early auctions, aiming for the asks of the cheapest gencos, instead of bidding at the equilibrium price. Our broker anticipated that this strategy, or similar variants, would be dominant among competitors. However, such strategies expose an opportunity for other brokers to make a profit by reselling energy, as we will explain below.

The Wholesale Bidding Strategy of cwiBroker. As a reference to the clearing process in the first auction for a specific timeslot we include Fig. 1a. Here the green lines (with open diamonds) indicate asks, sent in by the gencos, and the red lines (with closed circles) represent bids. The market clears at the intersection, where the limit prices of the asks and bids meet. 


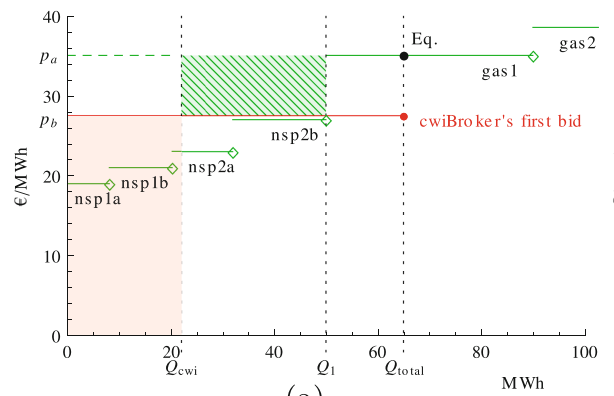

(a)

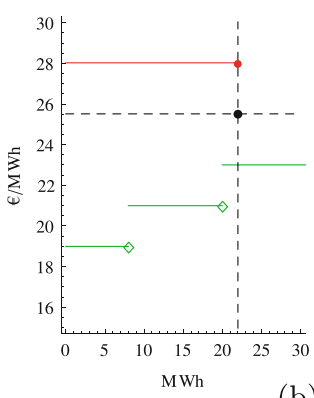

(b)

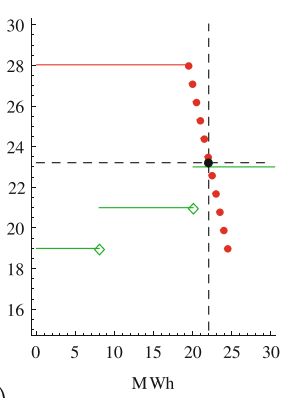

MWh

Fig. 1. (a) Our main bidding strategy. The green lines (with open diamonds) represent the asks of the gencos (subscripted with their names). In the first auction, we place a bid (cwiBroker's first bid) for a quantity $Q_{\text {total }}$. The limit price of our bid, $p_{b}$, is chosen just above the limit price of the last genco that has to operate fully to meet this demand (in this case nsp2b). When selling energy in future auctions, we ask a limit price, $p_{a}$, just below the next cheapest genco (gas1). The net price we pay is represented by the red area minus the green area. (b) Sending in multiple bids (red lines with circles). Compare the left situation (ordinary), to the right (including multiple bids). Due to the additional bids, the clearing price will be lower without otherwise influencing the outcome of the auction (Color figure online).

The first step in our bidding strategy is to estimate the equilibrium price and equilibrium quantity for the particular timeslot. Therefore, we estimate how much energy is going to be needed to fulfil the needs of all customers present in the tariff market (irrespective of their subscriptions). For this quantity, denoted $Q_{\text {total }}$, we depend on the prosumption estimator (see Sect.5). If all wholesale energy were to be traded in a single auction, then the equilibrium would be set at $Q_{\text {total }}$, at the price level of the genco's ask that intersects with this quantity (the point labelled 'Eq' in Fig. 1a). We expect that any energy that can be obtained below this price, can be resold in future auctions at a higher price.

Therefore, in the first auction our broker makes a bid for $Q_{\text {total }}$. However, the limit price will not be chosen at the equilibrium price, but below it. We choose our limit price $\left(p_{b}\right)$ just above the limit price of the second last genco that sells energy at the equilibrium. This is the last genco that has to operate fully to meet the demand of $Q_{\text {total }}$. In the situation in Fig. 1a, this is genco 'nsp2b'.

The actual energy obtained in the first auction is denoted $Q_{1}$. It is possible that $Q_{1}$ is larger than the energy needed to meet the total demand of the customers of cwiBroker (denoted $\left.Q_{c w i}\right)$. In this case, the difference $\left(Q_{1}-Q_{c w i}\right)$ can be sold again in the 23 remaining auctions for this timeslot. For these auctions, the gencos from whom we have bought power in the first auction will no longer submit asks. Therefore, if we set the limit price of our asks, $p_{a}$, to just below the limit price of the next cheapest genco (at the estimated equilibrium price), this will be the lowest ask price. It will however be higher than the price we paid for it ourselves $\left(p_{a}>p_{b}\right)$. If other brokers then want to buy wholesale power, they will have to bid at a limit price of at least $p_{a}$. The difference in unit price $\left(p_{a}-p_{b}\right)$, 
multiplied by $\left(Q_{1}-Q_{c w i}\right)$ is the revenue for our broker. This is represented by the green (hatched) area in Fig. 1a. The red (filled) area represents our costs. In some cases, the green area may become larger than the red area, and thus it is possible to make a net profit on the wholesale market, even when buying energy. This is indicated by a negative unit price.

Summarizing, we derive benefit from the opportunity to buy energy below the equilibrium price in the first auction. In the remaining auctions, we try to earn back some of our investments by sending in asks at the equilibrium price. If we do not succeed in buying enough energy in the first auction, we fall back to another strategy. This other strategy is to simply look at the orderbook (the set of uncleared orders of the previous auction) and bid for more energy just above the limit price of the last uncleared ask.

Our main strategy, described above, can easily be countered by other brokers. The other brokers could for example mimic our strategy. If they also bid on large quantities in the first auction, but at a slightly higher limit price, their bids will get cleared, and ours rejected. To solve this, we could, in turn, raise our limit price slightly above the competitor's price. However, at a certain point we will reach the price level of the next cheapest genco. Above this price level, our broker risks buying more energy than it can ultimately sell again, because now both our bid and the competitor's bid may be accepted. In fact, at this point we will have reached the equilibrium price and quantity. We conclude that our strategy can only fare well in the absence of competition in the first auction.

Multiple Orders per Auction. We note that there is no limit on the number of orders brokers can submit for a single auction. It can be advantageous to send in multiple orders, as we will explain below. If we send in bids, we want the market clearing price to be as low as possible, and if we send in asks, we want it to be as high as possible. Suppose we want to buy an amount $q$, at limit price $p$. We then send in the usual bid $(q, p)$, but now we also send in a few other bids, with a very small quantity, and each with a slightly lower unit price. So we send in the following bids as well:

$$
\left\{\left(q_{\epsilon}, p-i \Delta_{p}\right) \quad \mid \quad i \in\{1, \ldots, N\}\right\}
$$

where $q_{\epsilon}=2^{-1074}$ (the lowest possible value for a double in Java), for some $\Delta_{p}$ and some $N$. We used $N=8$, not too high in order to limit overhead for the server. $\Delta_{p}$ was calculated from $p$ and $N$ such that it would yield reasonable step sizes. If our original bid is cleared, it is likely that some of these additional bids will get cleared too, up to the last one with a limit price much closer to the highest ask price (see Fig. 1b). Effectively, we make sure the clearing price is as close as possible to the limit price of the last cleared ask. Note that this only works if the intersection of asks and bids occurs such that the last cleared ask (rather than the last cleared bid) is partially executed. Similarly, when selling energy, it can be advantageous to send in multiple asks, in order to raise the clearing price to a value as close as possible to the last cleared bid. 


\section{Prosumption Estimator}

In order to make optimal decisions broker agents must be able to predict the total portfolio prosumption of a timeslot. This is the portfolio production minus the portfolio consumption. Our broker computes the estimation $\hat{Q}_{t}$ of the portfolio prosumption in timeslot $t$ in the following way:

$$
\hat{Q}_{t}=\sum_{c \in C} \sum_{\tau \in T} n(c, \tau) \hat{q}_{c, h(t)}(t e m p(t))
$$

$C$ is the set of customer types, $T$ is the set of tariffs published by our broker, $n(c, \tau)$ is the number of customers of type $c$ subscribed to tariff $\tau$, and temp $(t)$ is the predicted temperature in timeslot $t . h(t)$ is the hour of the week of timeslot $t$, which is composed of the hour of the day (0-23) and the day of the week (weekday, saturday, sunday). The predicted prosumption $\hat{q}_{c, h}(x)$ per customer of type $c$, for hour of the week $h$, and temperature $x$ is given by

$$
\hat{q}_{c, h}(x)=a_{c, h}+b_{c, h} x
$$

After every timeslot $t$, the parameters $a_{c, h}, b_{c, h}$ for hour of the week $h=h(t)$ and all customer types $c$ are updated using the data observed so far. First, the observed prosumption per customer for each type $c$ and the observed temperature are added to the data. Then, a least squares fit is performed on the data to compute $a_{c, h}$ and $b_{c, h}$. In the Power TAC in 2013 the prosumption estimator only used temperature as a predictor, but in future versions we will incorporate other weather conditions as well.

\section{Tournament 2013 Results}

Our broker ended second out of seven participants in the competition. It obtained the highest score in each of the 7-player games and in five of the six 2-player games it played. In the 4-player games, cwiBroker always had a decent score (six times 1st, 11 times 2nd, three times 3rd). Furthermore, cwiBroker had a positive score in all games except one (the 4-player game with game number 120, where all players had a negative score). See Table 1 for the scores of all brokers in each setting.

\subsection{Tariff Market}

In duopoly games we played a strategy that copied the opponent's tariffs. A nice example of where it was profitable to do this, is the game against INAOEBroker02. This broker did not obtain large market shares in games against other brokers (see Table 2), because its tariffs were not very competitive. Although our broker obtained a market share of only 0.47 , our profit against INAOEBroker02 was much larger than any other broker, even though they obtained market shares of at least 0.89 . 
Table 1. Total scores per category per broker. The normalized values represent the z-scores. The total normalized scores define the competition results.

\begin{tabular}{l|r|r|r|r|r|r|r}
\hline \multirow{2}{*}{ Broker } & \multicolumn{2}{l}{ Score } & \multicolumn{2}{l}{ Normalized } \\
\cline { 2 - 9 } & \multicolumn{1}{l|}{ 7-player } & 4-player & 2-player & 7-player & 4-player & 2-player & total \\
\hline TacTex & $-705,248$ & $13,493,825$ & $17,853,189$ & 0.386 & 0.449 & 0.691 & 1.526 \\
\hline cwiBroker & 647,400 & $12,197,772$ & $13,476,434$ & 0.437 & 0.442 & 0.536 & 1.415 \\
\hline MLLBroker & 8,533 & $3,305,131$ & $12,796,064$ & 0.413 & 0.391 & 0.395 & 1.199 \\
\hline CrocodileAgent & $-361,939$ & $1,592,764$ & $8,336,061$ & 0.399 & 0.381 & 0.311 & 1.091 \\
\hline AstonTAC & 345,300 & $5,977,354$ & $5,484,780$ & 0.425 & 0.406 & 0.254 & 1.086 \\
\hline Mertacor & $-621,040$ & $1,279,380$ & $4,919,087$ & 0.389 & 0.380 & 0.234 & 1.003 \\
\hline INAOEBroker02 & $-76,112,159$ & $-497,131,383$ & $-70,255,037$ & -2.449 & -2.449 & -2.421 & -7.319 \\
\hline
\end{tabular}

Table 2. The market share of each broker in duopoly games. Market share is calculated as the normalised net consumption of a broker's portfolio (negative numbers indicate net production of a brokers customers). The final column represents the mean $( \pm$ standard-deviation) of the market share of a broker.

\begin{tabular}{l|l|l|l|l|l|l|l|l}
\hline & Aston & Croc & cwi & INAOE & Mert & MLL & Tex & $\mu \pm \sigma$ \\
\hline AstonTAC & - & 0.95 & 0.98 & 0.89 & 1.03 & 0.99 & 0.66 & $0.78 \pm 0.37$ \\
\hline CrocodileAgent & 0.24 & - & 0.54 & 1.18 & 0.77 & 0.00 & 0.26 & $0.43 \pm 0.43$ \\
\hline cwiBroker & 0.02 & 0.46 & - & 0.47 & 0.89 & 0.71 & 0.00 & $0.36 \pm 0.36$ \\
\hline INAOEBroker02 & 0.11 & -0.18 & 0.53 & - & 0.01 & 0.10 & -0.15 & $0.06 \pm 0.24$ \\
\hline Mertacor & 0.18 & 0.36 & 0.11 & 0.98 & - & 0.77 & 0.09 & $0.36 \pm 0.38$ \\
\hline MLLBroker & 0.01 & 1.00 & 0.29 & 0.90 & 0.23 & - & 0.00 & $0.35 \pm 0.43$ \\
\hline TacTex & 0.52 & 0.95 & 1.00 & 1.15 & 1.09 & 1.00 & - & $0.82 \pm 0.41$ \\
\hline
\end{tabular}

In the games against AstonTAC and TacTex, our broker did not attract many customers, because their strategies were too competitive. TacTex was able to benefit from this. AstonTAC however, was unable to make a profit, although it had almost all customers.

In oligopoly games, our broker attempted to estimate the optimal tariff price using subscription models. In most games, however, our broker overestimated the number of subscriptions of its tariffs. As a result, the estimated optimal tariffs were too expensive. Fortunately, the back-up strategy (decreasing the tariff price, until customers subscribed to the tariff) ensured we still had some customers in these games.

\subsection{Wholesale Market}

We analysed the results of the Power TAC wholesale market, to validate whether our strategy was successful. We investigated how much energy every broker bought or sold, and how much money it paid or received for this. In this section, we will represent the aggregated outcomes of the 24 relevant auctions, for each timeslot $t$ and each broker $b$, as a total quantity $Q_{t, b}$ and a total cost $C_{t, b}$. 


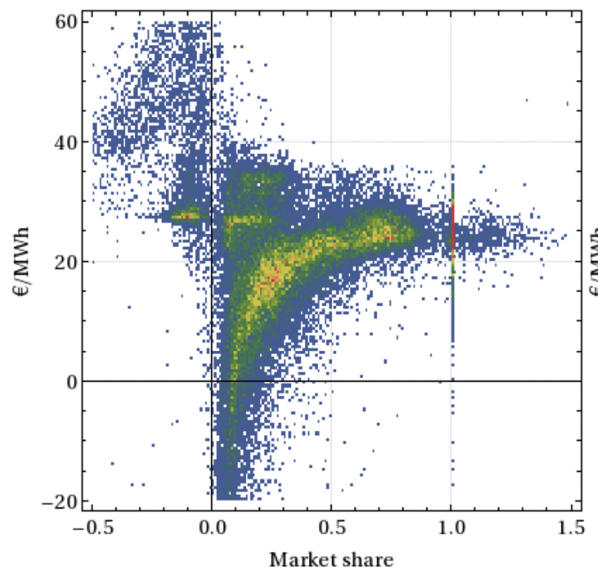

(a) cwiBroker

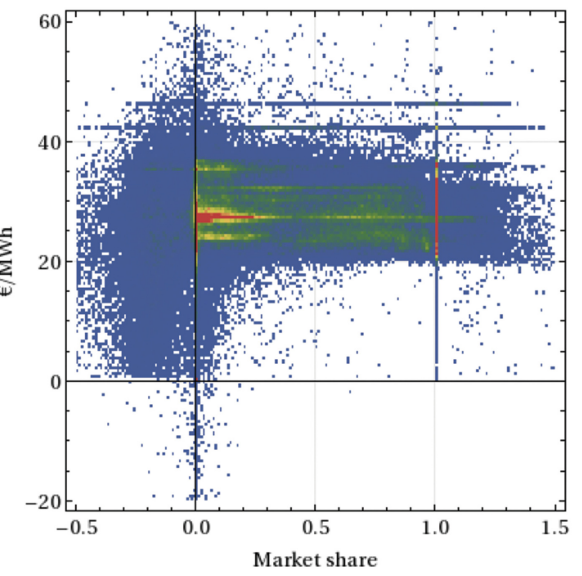

(b) Other brokers

Fig. 2. Results of the wholesale market for cwiBroker and other brokers (all brokers except cwiBroker) combined. The yellow and red colors represent higher densities of points (Color figure online).

Figure 2 shows the results for all timeslots for all games that were played in the competition as a heat map for cwiBroker and, for comparison, also for the other brokers. The vertical axis represents the unit price $\left(C_{t, b} / Q_{t, b}\right)$ and the horizontal axis a market share $\left(Q_{t, b} / \sum_{i \in B} Q_{t, i}\right.$, where $B$ is the set of all brokers). The market share is a normalised measure for the net amount of energy a broker bought or sold, relative to the total of all brokers. The market share may be negative (indicating the broker has sold energy and the combined market share of the other brokers was positive) or larger than 1 (indicating the broker bought energy and the combined market share of the other brokers was negative).

From the results in Fig. 2, it becomes clear that for market shares between 0 and 0.5 , cwiBroker often obtained a much lower unit price than its competitors. The unit prices for cwiBroker are generally below 20€/MWh, while for other brokers this is the lower limit. Negative unit prices are also present, which effectively means money was earned even when we had to buy energy. Furthermore, for negative market shares, the unit prices for cwiBroker are much higher than those of other brokers. This means our broker earns more money by selling energy than other brokers. From this we conclude that our strategy was very successful for market shares below 0.5. This is the case when the total energy needed by other brokers is larger than that of cwiBroker. Our resaling strategy ensured that the other brokers had to buy part of the energy they needed from our broker. For market shares above 0.5, there is less opportunity to make a profit by trading in a smart way, but our broker still obtained energy at unit prices comparable to, or lower than other brokers.

The results of the wholesale trading in the 7-player setting are of particular interest, since in this setting all brokers compete directly with each other. 
We aggregated the outcomes of all auctions for all timeslots. This yields a total quantity and a total cost of energy on the wholesale market, per game per broker, which can again be converted to a unit price. The net unit price for wholesale energy of our broker ranges from 12.2 to $18.5 € / \mathrm{MWh}$. This is much lower than all other brokers, who pay at least $25.7 € / \mathrm{MWh}$.

Estimated Wholesale Earnings Indicator. We are interested in the relative importance of the wholesale market, compared to other incomes and expenses in the competition. Therefore, we estimate for each game how much money brokers spent on wholesale energy compared to others. For each game, we made a least squares linear fit through the data $\left(Q_{t, b}, C_{t, b}\right)$ of all timeslots in this game, for all brokers except one (the broker for which we built the model to compare with). This yields coefficients $a$ (in $€ / M W h$ ) and $b$ (in $€$ ) in a model of the average costs $C$ for a specific amount of wholesale energy $Q$ for competitors:

$$
C=a Q+b
$$

The difference between the costs according to the model and the actual costs then indicates how much money a broker saved (or spent excessively) compared to competitors in this game:

$$
E_{b}=\sum_{t \in T}\left(a Q_{t, b}+b-C_{t, b}\right)
$$

where $E_{b}$ is an indication for the wholesale earnings (in $€$ ) of the broker compared to its competitors. $T$ is the set of all timeslots in a game. The total values (summed over all games in a category) for each broker for each type of game can be found in Table 3 . If we compare these values with the competition results in Table1, we can see that a significant part of our total profit is due to our wholesale strategy. For the 7-player games, the relative earnings in the wholesale market are even larger than our total profit in these games.

Table 3. An indicator for the performance of each broker in wholesale trading. The values represent the amount of money (in €) a broker saved (or spent excessively, in case of negative numbers) on wholesale energy, compared to competitors. The scores represent the total amount of money for a broker for all games in a category.

\begin{tabular}{l|r|r|r}
\hline Broker & \multicolumn{1}{|l|}{ 2-player } & 4-player & \multicolumn{1}{l}{ 7-player } \\
\hline AstonTAC & 924,200 & 770,100 & 1,100 \\
\hline CrocodileAgent & $-1,302,000$ & $-2,001,900$ & $-101,200$ \\
\hline cwiBroker & $2,456,000$ & $4,332,600$ & 835,700 \\
\hline INAOEBroker02 & $-3,926,700$ & $-2,176,500$ & $-318,700$ \\
\hline Mertacor & $-3,675,800$ & $-519,300$ & $-20,000$ \\
\hline MLLBroker & $-1,700,300$ & $-848,100$ & $-110,400$ \\
\hline TacTex & $1,011,200$ & 420,000 & $-1,177,400$ \\
\hline
\end{tabular}


We also checked the relative advantages of sending in multiple orders for an auction. In order to estimate how much money brokers could have saved, we analysed the wholesale result for every auction in the competition. If the orders in each auction are augmented with additional orders with infinitesimal small quantities, as described in Sect. 4, the clearing price will be either higher or lower, but the cleared quantities will remain the same. In case all submitted wholesale orders were augmented, cwiBroker would have made less money (€502,771 less over the entire competition), because the price at which we sold energy would have been lower. Other brokers could have saved on average $€ 363,494$ per broker, summed over all games. The potential benefits of this strategy are therefore not that large, compared to the total income and expenses of brokers. It should however be noted that this strategy is completely free of risks.

\subsection{Prosumption Estimator}

The prosumption estimator must predict the total portfolio prosumption as accurately as possible. In order to measure its performance we define a performance measure for the relative absolute error $\operatorname{err}_{g, t}^{r e l}$ in game $g$ for timeslot $t$ :

$$
e r r_{g, t}^{r e l}=\frac{\left|Q_{g, t}-\hat{Q}_{g, t}\right|}{Q_{g, t}^{\text {prod }}+Q_{g, t}^{c o n s}}
$$

$Q_{g, t}$ represents the actual prosumption and $\hat{Q}_{g, t}$ the estimated value, one timeslot ahead. $Q_{g, t}^{\text {prod }}$ and $Q_{g, t}^{\text {cons }}$ represent respectively the production and the consumption of cwiBroker's customers ( $\operatorname{such}$ that $Q_{g, t}=Q_{g, t}^{\text {prod }}-Q_{g, t}^{\text {cons }}$ ). We divide by the sum of production and consumption, rather than their difference, because we are interested in the error relative to the size of our total portfolio, rather than to the net consumption. Additionally, in case $Q_{g, t}^{\text {prod }}$ and $Q_{g, t}^{\text {cons }}$ have similar values, we would otherwise risk dividing by zero.

Note that $\operatorname{err}_{g, t}^{r e l}$ for a single timeslot $t$ typically decreases during a game, because the prosumption estimator improves its model by learning from the game data. We are not only interested in its performance at the end of the game, though, as a prosumption estimator must perform well during the entire game. Therefore, we computed $\operatorname{err}_{g}^{r e l}$ for an entire game $g$ as the mean of the relative prosumption errors $\operatorname{err}_{g, t}^{r e l}$ over all timeslots in game $g$. Over the entire competition, the mean of $\operatorname{err}_{g}^{r e l}$ ( \pm standard-deviation) is $0.131 \pm 0.053$.

Our prosumption estimator used temperature as a predictor. However, production depends on cloud cover, wind speed, and wind direction, rather than temperature. We had not yet implemented this in the 2013 competition, but we expect that it could have improved our prosumption estimator even further. In order to analyse this, we also tested our prosumption estimator offline. In this experiment we ran 30 simulations, in which we applied three different prosumption estimators to the same portfolio. As a benchmark we used the prosumption estimator of the sample broker [7], which is delivered as part of the Power TAC 
platform. Furthermore, we used the prosumption estimator from the 2013 competition, and an improved version, which used cloud cover and wind speed instead of temperature as predictors for solar and wind energy respectively.

We computed the relative prosumption estimation error err $_{g}^{r e l}$ for each prosumption estimator. The means $( \pm \mathrm{sd})$ are $0.151 \pm 0.037$ for the sample broker, $0.107 \pm 0.029$ for the 2013 version, and $0.089 \pm 0.021$ for the improved version. In each simulation, the 2013 prosumption estimator was better than the sample broker's, and the improved version was never worse than the 2013 version.

\section{Conclusion}

We developed cwiBroker for the 2013 Power TAC.

The duopoly tariff market strategy was inspired by TFT in the IPD game, which generally resulted in good performance. Unfortunately, the oligopoly tariffmarket strategy was often not able to reliably predict the optimal tariff to publish, and resulted in tariffs that were too expensive to attract any customers. However, the back-up strategy of decreasing our tariff price until a satisfactory number of customers was obtained solved this issue to some degree.

On the wholesale market, cwiBroker was able to buy energy for bargain prices in the first auction for each timeslot. In most cases, it was able to sell the surplus of energy in later auctions for a higher price. The result was that our broker was able to make significant profits, by trading on the wholesale market. From the 7-player games we can conclude that, when competition on the tariff market is fierce, it is essential to have a good wholesale strategy. We achieved the highest scores in each of the 7-player games, where it was very hard to earn money on the tariff market. Our wholesale strategy ensured we still made a net profit.

Finally, our broker was able to accurately predict the prosumption of its customers. This was essential input for our wholesale strategy, and in the estimation of the profitability of tariffs that our broker considered. The combined set of strategies resulted in a versatile and robust broker, that performed very well in all settings that were tested, and ended second overall in the competition.

\section{References}

1. Schweppe, F.C., Caramanis, M.C., Tabors, R.D., Bohn, R.E.: Spot Pricing of Eleciricity. Kluwer Academic Press, Boston (1988)

2. Stoft, S.: Power System Economics: Designing Markets for Electricity. Wiley-IEEE Press, Hoboken (2002)

3. Ketter, W., Collins, J., Reddy, P., de Weerdt, M.: The 2013 Power Trading Agent Competition, ERIM report Series Reference No. ERS-2013-006-LIS (2013)

4. Axelrod, R., Hamilton, W.D.: The evolution of cooperation. Science 211(4489), 1390-1396 (1981)

5. Ketter, W., Peters, M., Collins, J.: Autonomous agents in future energy markets: the 2012 power trading agent competition. In: Association for the Advancement of Artificial Intelligence (AAAI) Conference, Bellevue (2013) 
6. Harris, L.: Trading \& Exchanges: Market Microstructure for Practitioners. Oxford Press, Oxford (2003)

7. Ketter, W., Collins, J., Reddy, P.: Power TAC: a competitive economic simulation of the smart grid. Energy Econ. 39, 262-270 (2013) 